\title{
Text verification instruction manual using the ontologies
}

\author{
A.A. Orlova \\ Department construction and design of the aircraft \\ Samara State Aerospace University \\ Samara, Russia \\ a.aorlova@yandex.ru
}

\author{
N.M. Borgest \\ Department construction and design of the aircraft \\ Samara State Aerospace University \\ Samara, Russia \\ borgest@yandex.ru
}

\begin{abstract}
The paper deals with the verification of manuals using ontologies built using ontological editor Fluent Editor. The ontological editor Fluent Editor creation of ontologies by using Controlled Natural Languages $(\mathrm{CNL})$, which is close to a natural language that simplifies and facilitates the user's work. Text conversion algorithm manuals of natural language in a controlled. Based on the construction and visualization of ontology instruction manuals, conclusions are drawn about the possibility of using ontological editor Fluent Editor as a tool to verify the completeness, uniqueness and comprehensiveness manuals.
\end{abstract}

Keywords - ontology; verification; instruction manual; Controlled Natural Languages; ontological editor

\section{INTRODUCTION}

Currently, the creation of manuals is carried out in special programs such as Help\&Manual, Dr.Explain and others [1, 2]. Specialists carry verification of created manual, as a rule, out using automated tools various competent in specific subject areas. The lack of strict formal rules in the organization of coordinated work in the preparation of documents may lead gives rise to all sorts of ambiguities, errors and omissions in the guidelines, which is unacceptable in the operation, assembly and use of any product or parts of product.

One way of innuendo and inaccuracy of solving problems such as the text is to present them in the form of an ontology. Visual design methods ontologies contribute to a more rapid and complete understanding of the structure of knowledge of any subject area, which is invaluable when checking the quality of the knowledge described. Accordingly, in this paper we propose to use to create the user guide is one of the software tools designed specifically for the design, editing and analyzing ontologies - ontological editor [3].

\section{BUILDING AN ONTOLOGY MANUALS}

\section{A. Selecting a tool for verifying the manuals}

Fluent Editor, which is used to create a ontology Controlled Natural Language. In order to solve the above problem ontological editor has been selected. In Fluent Editor controlled natural language is English. It is through the use of CNL, Fluent Editor allows you to build an ontology people who are not professionals in the field of information technologies, which is convenient for use editor specialists in the manufacturing sector [4].
The important point is the definition of "instruction manual" (IM). In this paper, under the IM refers to a document containing information about the structure, principle of operation, characteristics (properties) of the product. Its component parts and instructions necessary for proper and safe use of the product (intended use, maintenance, storage and transportation) and evaluation of its technical condition in determining whether to send it in for repair, as well as information on the disposal of the product and its components [5].

\section{B. Algorithm verification of manuals}

IM developed manufacturing facility in accordance with applicable standards, establishing rules for preparation, execution and registration of the design documentation, based on which is cost and ontology IM.

Development of IM should be carried out at the stage of preproduction process, and attached to all types of products coming into circulation in the market of the country [6].

Thus, there is a need to develop test methods created and produced by IM. Ontology instruction manual should be created, based on the standards and examples of already existing guidelines. Implementation of the guidelines texts verification in this paper is carried out by means of visualization of the selected ontology editor. The algorithm for converting manual verification Fluent Editor represented in block diagram form in Fig. 1.

\section{Application of algorithm verification for the etching process instruction manual}

As an example, for developing an ontology metal etching process is selected. This process is the use of technological methods for the controlled removal of the surface layer of material from the work piece under the influence of specially selected chemicals. Etching includes grinding, polishing, followed by details of its location by the operator (human) in the first degreasing bath, and then etching in a bath, and in the end washing bath [7].

Before describing the instruction manual of the execution of the process, you must create an ontology of the etching process. Let the two performing activities subject «Human» and «apparatus-for-etching», further expanding the ontology describes the types «apparatus-for-etching» using «is a» relations, after which each of the actors involved in the process 
of etching, assign actions, that it needs to perform at a certain stage of the process (Fig. 2).

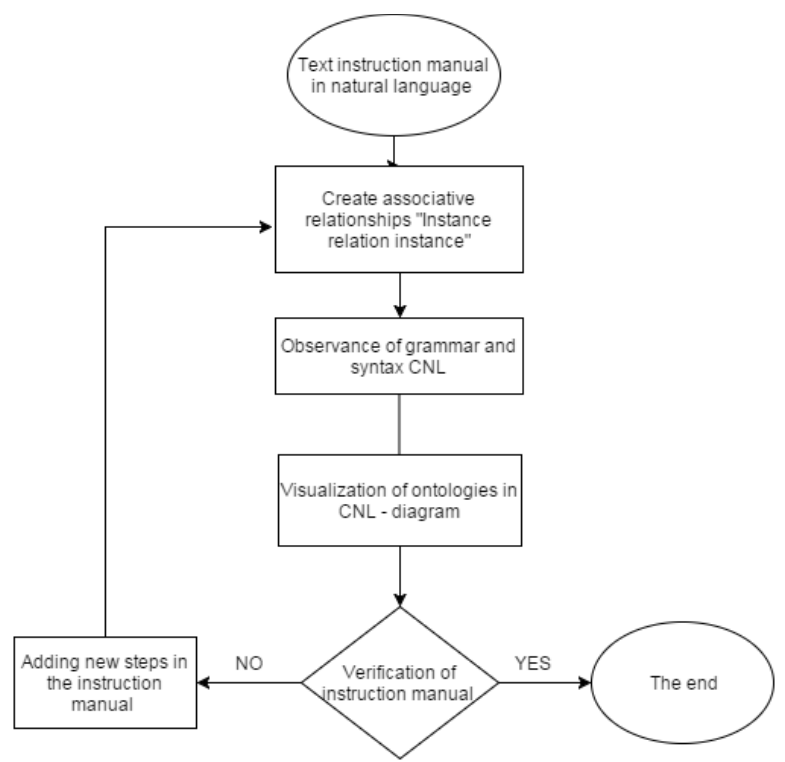

Fig. 1. Algorithm for converting text guidelines for verification in the ontological editor by CNL.

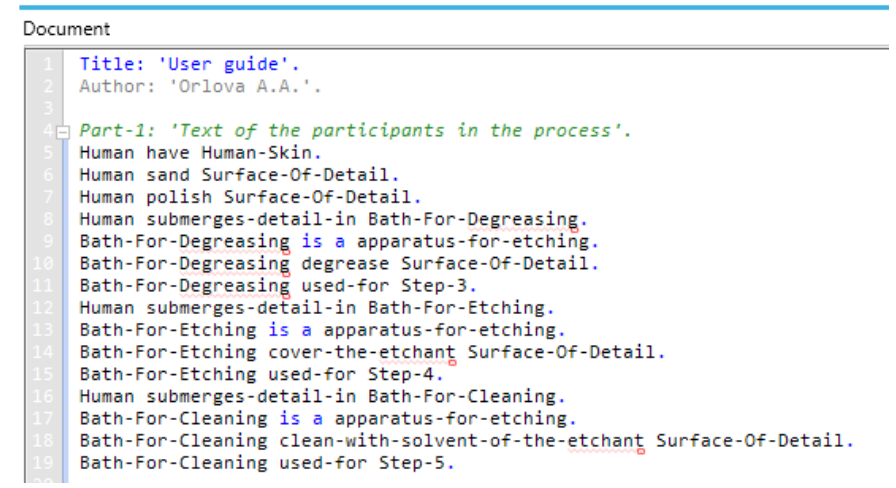

Fig. 2. Ontology of the etching process, presented at the CNL.

To demonstrate the developed algorithm modeled generalized IM fragment describing the sequential execution of the etching process, based on a study of examples of IM and their construction standards:

1. Sand the surface of detail

2. Polish the surface of detail.

3. Degrease the surfaceof detail.

4. Cover the surface of detail the etchant. ATTENTION! DO NOT ALLOW THE ETCHANT TO HUMAN SKIN.

5. Clean the surface of detail with solvent of the etchant and the etching products.

The relationship between the concepts in the IM, as a rule, are given non-hierarchical relationships and associative relationships, which are set at CNL-dependent «Instance relation Instance. », for example, «Step-1 sand Surface-Of-Detail.». The developer to implement ontologies correct sequence of the sentence in the CNL enters a copy of the «Step-1». Also, the introduction of additional copies of the «Step-1», «Step-2», etc. It allows instruction manual to submit proposals in the imperative form in compliance with the sequence of operations. Figure 3 shows the text of the manual after the job depending on «Instance relation Instance.».

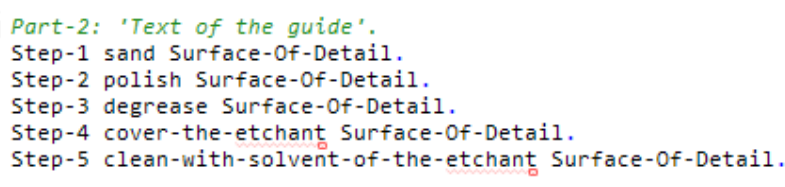

Fig. 3. The text of the manual after the job depending on «Instance relation Instance.».

Thus, using the above transformation is performed item 6.4 of GOST 2.601-95: "The text in presenting guidance on carrying out the work used a verb in the imperative mood, for example, «Open the door», «Press», etc.". Introduction copies «Step-1», «Step-2», etc. It helps carry a description of the performance of any work ontological model of manual in a logical sequence of their execution (item 6.5 of GOST 2.601-2013).

Follow these steps to comply with security warnings are described in the ontology. to introduce the ontology instance «Attention-1» for the implementation of security measures (Figure 4). Warnings on safety should be described in the Operating Manual, as there is a standard (item 6.7 GOST 2.6012013): "... in the development of documents, special attention should be paid to the account of the requirements for compliance with safety measures for maintenance and repair of products. The text of these requirements should be preceded by a warning word «Attention», etc"

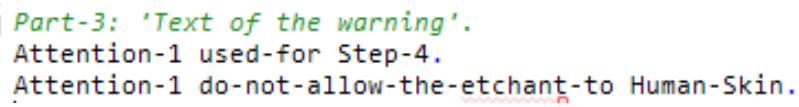

Fig. 4. Representation of warnings to comply with security measures in the form of ontologies on CNL.

\section{VERIFICATION OF INSTRUCTION MANUAL WITH THE HELP OF VISUALIZATION}

\section{A. Presentation of ontology as CNL-diagram}

Checking the completeness and correctness of the established instruction manual is carried out by visualization of ontology, namely, to create relationships and instances represented graphically by CNL-diagram (Figure 5). One skilled in the subject area will be easier to assess the correctness of the prepared guidance by imaging in CNL-diagram terms and relations between them.

Skilled investigated domain must carefully verify the visualization of ontology, the following sequence of actions:

1. Select one of the copies of the «Step-1», «Step-2», etc.

2. Continuation of checking relations between instances for each of the branches of the selected item 1 instance. Moving along the branches towards the object, the associated relationship, you can get a complete description of the action performed, including the implementation of the object and means of implementing the action performed. 


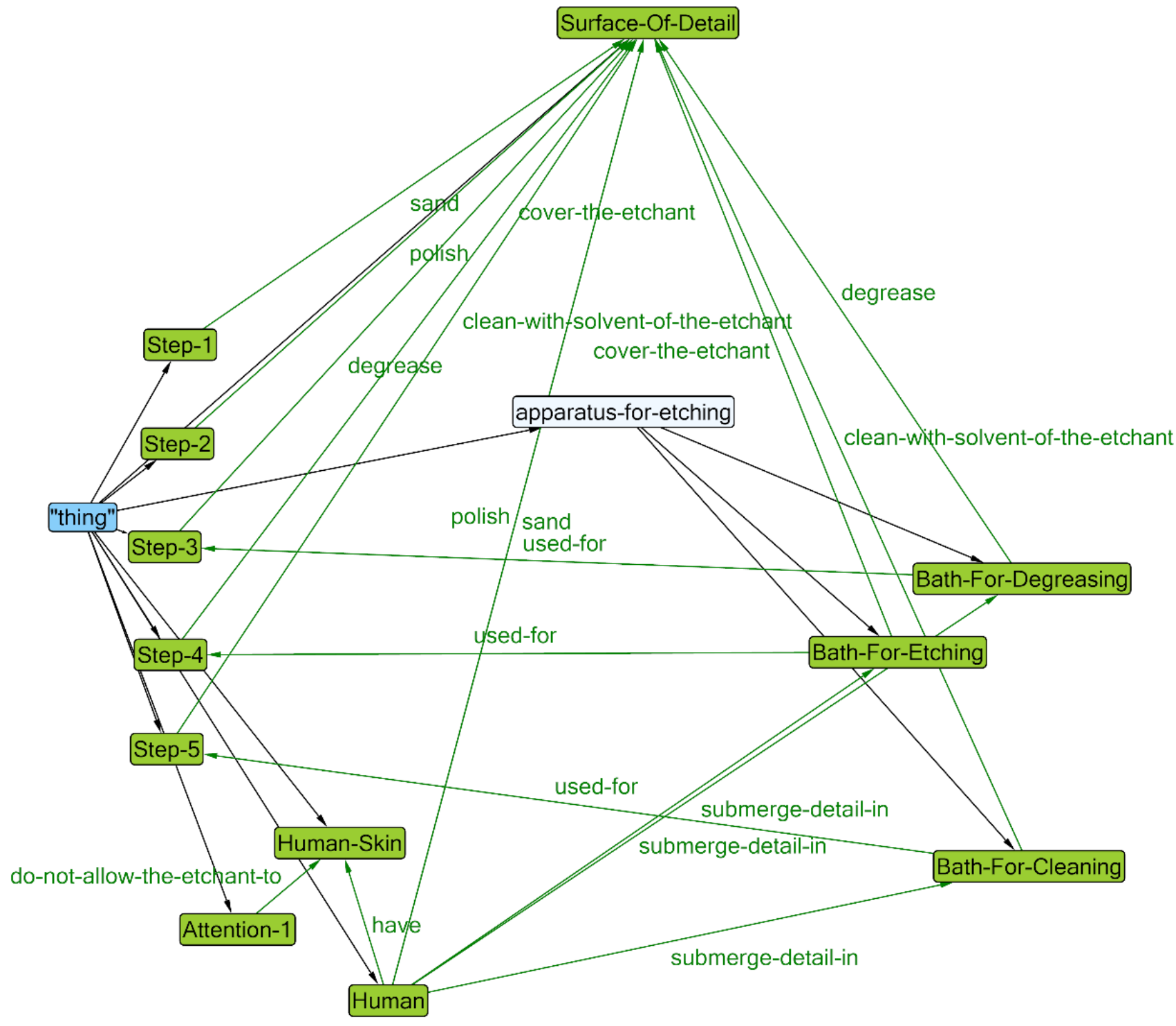

Fig. 5. CNL-diagram ontology process of etching.

3. Repeat 1 and 2 points for each instance of «Step- $1 »$, «Step$2 »$, etc. consistently performing verification of the relationship between objects and subjects of ontology related to each of the steps.

4. The conclusion of correctness of the instruction manual.

5. Supplement manual the necessary relationship and instances.

6. Verification amended guidance.

Check the completeness of instruction manual, as a rule, carried out by experts with sufficient knowledge in the field of development of the design and operational documentation for products able to competently and clearly outline the key moments in the process of use. Ontological editor Fluent Editor can serve as a tool to simplify the recording and verification guidelines.

\section{CONCLUSION}

Design manual conversion algorithm for verification easy and simple to use, its use does not take a lot of time with a specialist. Ontological editor Fluent Editor has the necessary mechanisms to verify user manuals such as ontology construction of CNL, visualization of associative relationships built ontology via CNL-diagram. All of these mechanisms can contribute to verify the operating instructions and to avoid possible errors and ambiguities of relations between the terms and incomplete of guide.

\section{REFERENCES}

[1] Help\&Manual, available at: http://www.helpandmanual.com/

[2] Dr.Explain, available at: http://www.drexplain.ru/ 
[3] Shustova D.V. Approach to developing a semantic basis of information systems for aircraft systems design and production // Ontology of designing, №1 (15), pp.69-84

[4] FluentEditor 2014, available at: http://www.cognitum.eu/semantics /Fluent Editor/

[5] GOST 2.601-2013. Unified system for design documentation Operational documents. - Moscow: Publishing House of Standards, 2013. $-36 \mathrm{p}$.

[6] Guidelines for the development and preparation for the adoption of draft technical regulations: the Federal Law of December 27, 2002 № 184 "On Technical Regulation" // Meeting of the legislation. - 2006. - 204p.

[7] M. Beckert, H. Klemm. Methods metallographic etching / Reference book. - Moscow: Metallurgy, 1988. -400p. 
